algunas de las arrugas en fachada desaparecen al bajar las persianas, los escaparates cambian de aspecto con los cambios de estación. La huella que despliega el comercio a ras de suelo convierte la ciudad en cinética -porque está constituida por elementos también en movimiento (Mehrotra, 2005)os paisajes a ras de suelo se muestran variables en su configuración física, vibran se mueven, cambian se rehacen; y con esa transformacion constante, y se rehacen; y con esa transformación consta

Es, como explican Pannerai y Mangin (1999), una suma de tiempos cíclicos desiguales en conflicto constante con otros ritmos lineales que marcan las actividades rutinarias de los ciudadanos. La carga $y$ descarga de productos frescos en mercadosy y descarga de productos frescos en mercados y supermercados a pimeras despliegue de las terrazas de bares y cafeterías, levantar las rejas y encender las luces, la música que acompaña la apertura de los establecimientos, los olores de las cocinas de los restaurantes, los primeros peatones cargados con bolsas: son signos sensibles a los sentidos que hacen tomar conciencla del tiempo cuando se camina la calle.

La presencia del movimiento del comercio evidencia un paso irreversible del tiempo que actúa sobre el paisaje urbano como lo hace sobre el paisaje natural, gastándolo, madurándolo y arrugándolo. El comercio genera parte de este repertorio de materiales esenciales que construyen la identidad urbana, que hacen visible en la ciudad superposiciones, mezclas y simultaneidades.

\section{BIBLIOGRAFÍA}

Clément, G. (2007), Le Jardin en mouvement : de la vallée au champ via le parc André-Citroën et le jardin planétaire, Paris: Sens et Tonka.

Cullen, G., (1961), El paisaje urbano: Tratado de estética urbanística. Barcelona: Blume, 1981.

Jacobs, J. (1961), Death and Life of Great American Cities, New York: Random House.

Lefebvre, H. (1992), "Visto desde la ventana", traducción de Élements de rythmanalyse a Martin, A. ed. (2014), La calle en 30 autores contemporáneos y un pionero, Barcelona: edicions UPC.

Mangin, D. (2004), Infrastructures et formes de la ville contemporaine La ville franchisée, Paris: Cerema

Mehrotra, R., (2005), Everyday urbanism : Margaret Crawford vs. Michael Speaks, Ann Arbor, Michigan University of Michigan, A. Alfred Taubman College of Architecture, New York: Distributed Arts Press.

Pannerai, P., Mangin, D. (1999), "Las calles corrientes", extracto del texto de Proyectar la ciudad, en Martin, A. (2014), La calle en 30 aut UPS co

Sansot, P. (2001), "Le flâneur ontologique”, Poïesis n. 12 La

Sansot, P. (1973), Poétique de la ville, Paris: Klincksieck. Simmel, G. (1903), "The Metropolis and Mental Life", en Bridge, G., i Watson, S. (eds.), The Blackwell City

Smithson, A., Smithson, P. (1960) "Fix: permanence and transience", en Architectural Review vol. 127-128 y consultado en Ordinariness and Light. Urban theories 1952-1960 and their application in a building project 1963 1970 (1990), Cambridge, MA: MIT Press.

ABSTRACT

A través de una calle recta dentro de la malla ortogona como la que ilustra el articulo se puede explicar cómo as tiendas que ocupan las plantas bajas modifican constantemente el aspecto y su relación con la calle y caracterizan el paisaje a ras de suelo, pese a la aparente alineación de fachadas y a la permanencia en la medida del parcelario. Las intervenciones de adicion, sustracción y modificación son intercambiables de un local a otro: cada tienda actúa para diferenciarse de las de al lado y asi conseguir captar la atención de los paseantes, y lo hace mediante estrategias que contribuyen a hacer que dentro y fuera se entrelacen y tomen las funciones los unos de los otros, colonizando el espacio urbano, pero también ensanchando la calle y convirtiendo el comercio en espacio colectivo.

PALABRAS CLAVE: Planta baja, comercio, Barcelona.

EULÀLIA GÓMEZ-ESCODA es arquitecta (ETSAB, 2004), doctora en Urbanismo (UPC, 2015) y profesora asociada del Departamento de Urbanismo y Ordenación del Territorio en la ETSAB. El artículo revisa parte de la investigación desarrollada por la autora para su tesis doctoral "Comerç, ciutat. Paisatges arran de terra", dirigida por el professor Enric Serra.

\title{
Un recorrido por los planos de suelo miesianos
}

\author{
Jordi Ros
}

Recibido 2015.12.08 :.: Aceptado 2015.12.10

DOI VERSION INTEGRA: 10.5821/palimpsesto.14.4684

A o largo de su trayectoria profesional Mies mantuvo una estricta observancia en relación a los dos

planos horizontales que acotaron sistemáticamente su arquitectura, el del suelo y el de la cubierta, al punto de que sus atributos formales así como la interrelación que establecen definen en gran medida su arquitectura.

De los dos, el plano de la cubierta ha sido el que ha suscitado un mayor análisis y reconocimiento: planas sin excepción, sus cubiertas han sido fruto de la evolución de unas tipologías estructurales, en hormigón y posteriormente en acero, que satisfacían una concepción de programa que priorizaba la flexibilidad. Contrariamente, el otro plano horizontal, el plano de suelo, ha sido menos explorado a pesar de su relevancia. Mies lo extiende mucho más allá de la proyección de sus respectivas cubiertas convirtiéndolo en espacio de mediación con el lugar y, a su vez, en espacio de transición desde el que construir el sistema de aproximación a su arquitectura.

En la mayor parte de casos, Mies dispuso sus planos de suelo a una cota algo elevada, sin que ni los requisitos topográficos del lugar ni las exigencias funcionales del programa lo requiriesen

Atendiendo a su continuada naturaleza masiva y extensa superficie, bien podrían ser denominados basamentos o podios. Sin embargo, la intensidad formal de las excepciones a esa doble condición, elevada y masiva, así como la reinterpretación radicalmente moderna con la que Mies trató el plano del suelo como parte de un complejo sistema relacional, aconsejaría desplazar el uso de esos términos hacia el de "plataforma", de acepción más abierta, y ello pese a que las primeras plataformas de Mies remitan claramente a los basamentos. [...] En ellas se reconoce la influencia de Behrens y particularmente de Schinkel, en aquel período de su arquitectura que le permitiría reforzar el lenguaje abstracto, y trabar la relación entre los dos estratos horizontales.

El regreso de Mies a Berlín, después del paréntesis de la primera guerra, lo vincularía intensamente con las emergentes vanguardias europeas, alejándolo al mismo tiempo del lenguaje Schinkeliano. Es el periodo de los denominados cinco proyectos que, de manera generalizada, se asocian con el inicio de la etapa moderna miesiana. Contrariamente, a efectos de la presencia y del sentido de la plataforma en esos cinco proyectos teóricos, realizados entre 1921 y 1924 difícilmente pueden aunarse.

Cronológicamente los tres primeros proyectos, los dos rascacielos de cristal y el edificio de oficinas de hormigón, a pesar de su indudable interés, no establecían mediación alguna con el terreno. Por el contrario, en los dos proyectos restantes, en la Casa de Ladrillo y especialmente en la Casa de Hormigón, la edificación descansa sobre un doble zócalo que se extiende por la figurada parcela, sobre la que el edificio se funde visualmente. $[\ldots]$

Cinco años más tarde, los tres proyectos, todos ellos relevantes, que Mies proyectaría en 1928, el Pabellón Alemán, la villa Tugendhat, y el menos reconocido de la remodelación de la Alexanderplatz, contenían plataformas, que se elevaban a diferente altura respecto al plano del suelo.

En Barcelona la plataforma del Pabellón perfiló su geometría y se erigió, por primera vez, en el auténtico solar del proyecto. La retícula del pavimento de travertino establecía con precisión sus límites y definía sobre ella una partitura sobre la que se dispondrían escrupulosamente estructura, cerramientos e, incluso, mobiliario. (FIG. 1)

La plataforma de la villa Tugendhat aportaría un nuevo registro, el sistema de acceso cedido al espacio vacío, allá construido entre el cuerpo principal y el de servicios, un espacio intersticial que volvería a presidir poco después el acceso de la casa Lange y, de forma magistral años más tarde el de la Farnsworth. [...]

Desde Alexanderplatz las plataformas urbanas de Mies se resolvían ajenas a los dictados de un tráfico, que de manera creciente empezaba a inferir equívocamente en la reflexión de los espacios urbanos contemporáneos. El Campus del IIT, el Federal Center, o la Mansion House londinense, serían ejemplares muestras de ello.

La ausencia de encargos durante los años treinta, con la ascensión del nazismo en la política alemana, comportó la desaparición significativa de las plataformas, ante la desaparición del lugar. Las propuestas teóricas, a menudo desarrolladas en el marco académico de la Bauhaus, que acabaría dirigiendo, renuncian a lugar, reproducen universos propios. De perímetro sistemáticamente rectangular, el recinto constituido por unos muros herméticos sustituía a las plataformas que soportaban las construcciones anteriores. Significativamente, la totalidad de las casas patio se desarrollarían a pie plano. No necesitaban desmarcarse de su entorno inmediato sencillamente porque no existía. Llegado este punto de depuración formal, e recinto amurallado, cual encofrado conceptual, podía ya ser retirado. Sería en gran medida gracias a las propuestas de las casas patio, tema que con variantes no abandonaría, que las plataformas de sus futuros proyectos adquirirían una condición más abstracta, más geométrica.

En el viaje que Mies emprendío a América, su arquitectura se desprendería de los muros que delimitaban sus propuestas. Por el contrario, la plataforma volvería a emerger como protagonista indiscutible de sus construcciones, definiendo los límites urbanos del conjunto de su etapa americana.

El primer encargo de Mies fue el del Campus de la IIT en Chicago. Durante los veinte años que estuvo al frente de Departamento de Arquitectura de aquella universidad, Mies construyó un gran laboratorio arquitectónico, primero en hormigón y ladrillo, luego en acero y vidrio.

El Campus del IIT, de 44 hectáreas de extensión emplazado en el Near South Side de Chicago, contenía dos plataformas, tan ejemplares como diversas: la de la propia ordenación general y la que construía el acceso de su Departamento de Arquitectura. Ambas representaban una excepción a la reiterada naturaleza de las mismas,
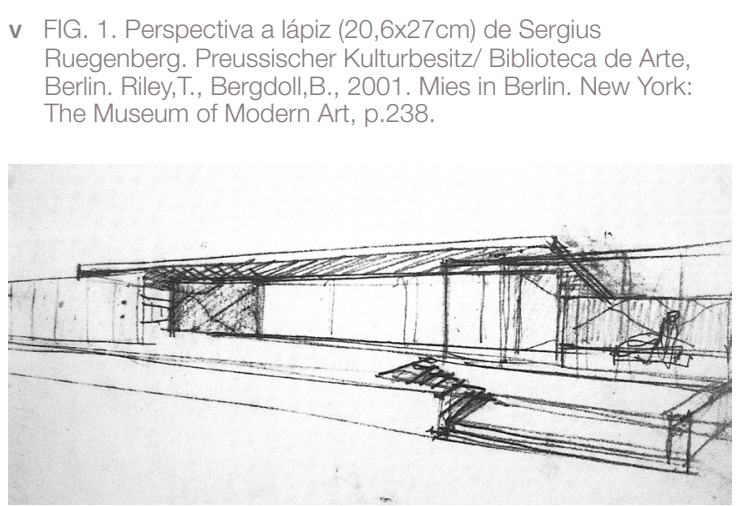
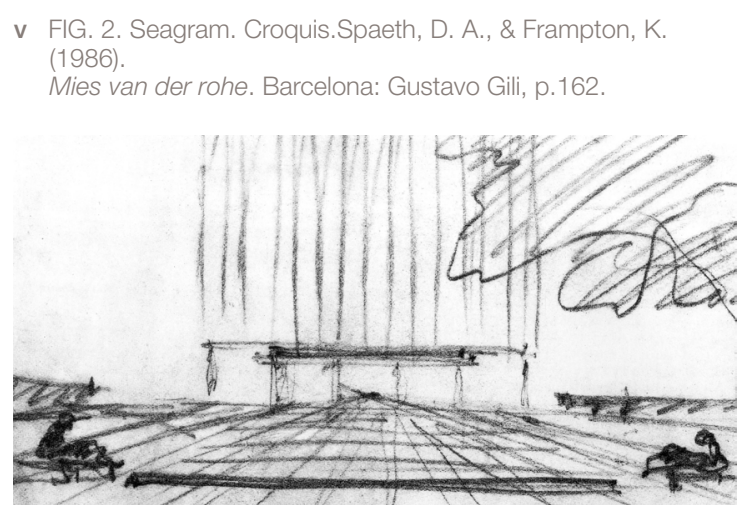

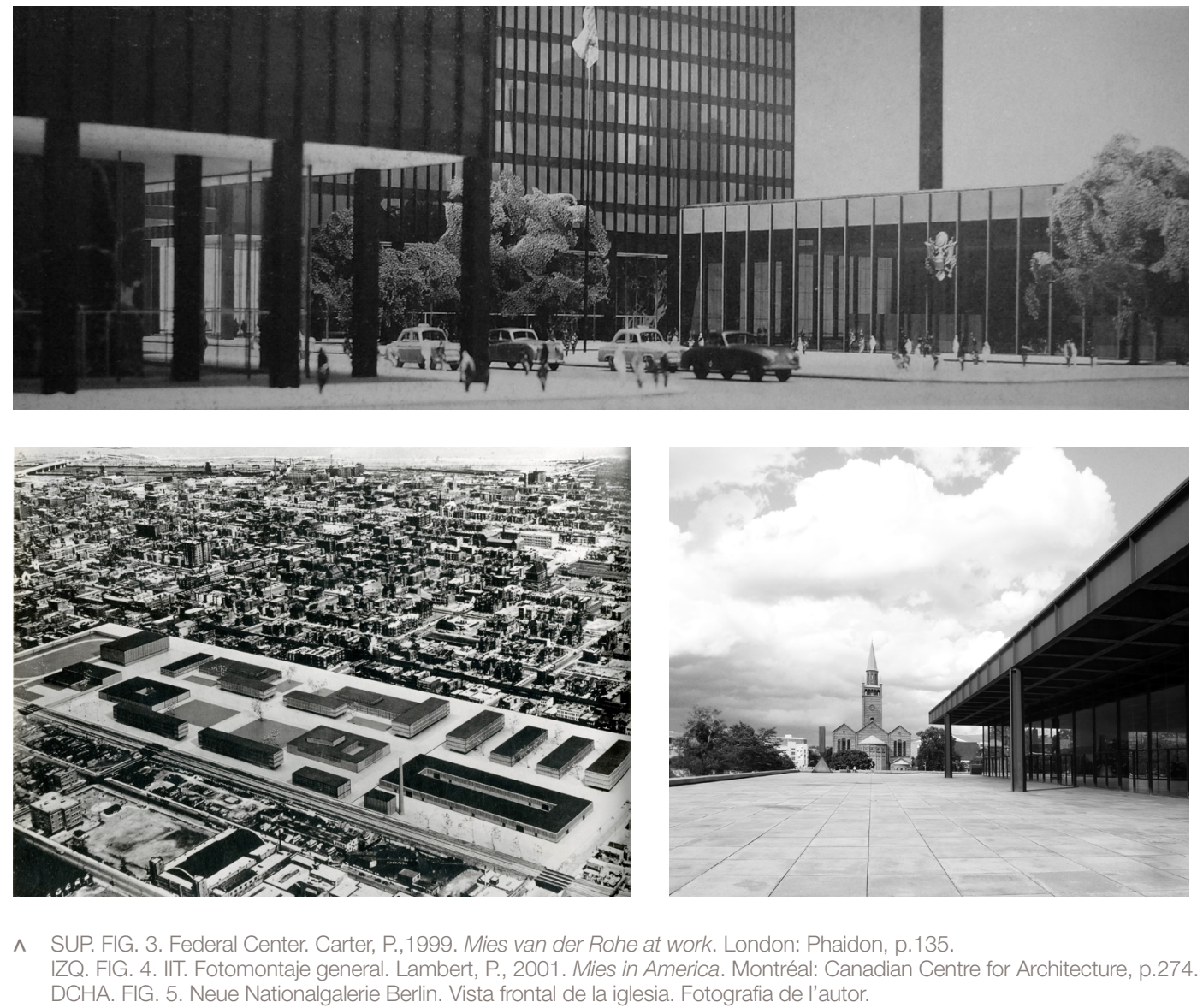

masivas y algo elevadas: En cuanto a la plataforma del conjunto, porque se hallaba a cota del terreno, respecto a la del Departamento de Arquitectura, en su última versión denominado Crown Hall, porque a pesar de estar elevada, no era masiva.

La plataforma de la ordenación del Campus destacaría por varios motivos, trascendentes en sus posteriores proyectos: - En planta, tanto por la transformación de las doce manzanas contenidas en el solar, seis en primera fase, en una única plataforma urbana, tan solo cruzada transversalmente por un vial intermedio, como por la introducción de una retícula ortogonal de 24 pies, primeramente respecto a las edificaciones, luego también respecto los espacios vacíos entre ellas. Esta estratégica unidad de medida devino un sistema normativo capaz de soportar una veintena de años y un número similar de proyectos sobre ella.

- En sección, documento significativamente inexistente, por las dudas que generó en Mies la manera en que los edificios debían depositarse sobre la plataforma.

En la primera versión del proyecto los dos edificios centrales descansaban directamente, sin mediación alguna, sobre el plano del suelo, mientras que los edificios perimetrales se elevaban porticadamente para favorecer la permeabilidad de sus plantas bajas. Por el contrario, en la segunda versión, que obligadamente incorporaba los viales intermedios inicialmente suprimidos, todos los edificios del campus, sin excepción, descansaban directamente sobre el suelo. Es decir, se habían eliminado todos los espacios porticados debajo de las edificaciones, lo que modificaba sustancialmente los respectivos sistemas de acceso y de circulaciones, así como el conjunto de visiones rasantes del proyecto.

El debate sobre si las edificaciones debían elevarse una planta o debían descansar directamente sobre el plano del suelo se mantuvo intensamente abierto a lo largo de todo el proceso de gestación de la propuesta, extendiéndose incluso en paralelo a la construcción de las primeras edificaciones. La profusión de perspectivas a mano del propio Mies, de una versión u otra, corroboraría tanto el interés como las dudas del arquitecto respecto a una decisión que resultó de capital trascendencia futura. La construcción en 1945 del primer edificio, el Navy Building, pareció sentenciar ese dilema con la supresión de la planta baja porticada. Sorprendentemente, la publicación, dos años más tarde, de un espléndido collage, con el lago Michigan al fondo, mostraba la escala de la sublime plataforma, y sobre ella, así como la diversidad de secciones de las edificaciones en planta baja, reflejo de las dudas que aún seguían acompañando a Mies en este episodio que, efectivamente, resultaría relevante. (FIG. 4)

Finalmente ningún edificio del Campus acabó ya elevando la planta baja, pero si atendemos a las obras posteriores podríamos convenir que la partida quedó en tablas: las futuras construcciones de baja altura descansarían masivamente sobre el suelo, disponiendo su acceso en el mismo plano de fachada mientras que las futuras construcciones en altura liberarían la planta baja ofreciendo el acceso bajo porche (los volúmenes del proyecto del Federal Center muestran ejemplarmente esa relación dual). (FIG. 3)

En 1947, un cuarto de siglo después de aquellos primeros rascacielos proyectados en Berlin, Mies retomaría, en la densa trama del downtomwn americano, el tema de las edificaciones en altura.

Si los alzados de los primeros rascacielos miesianos no tenían ni inicio ni final, eran un segmento de fachada de longitud indefinida que se depositaba indiferentemente sobre una fina línea abstracta, los rascacielos que Mies construyó en Estados Unidos cuidaban especialmente sus plantas bajas, entendidas como elemento de transición entre el edificio y la ciudad. [...]

Sin duda alguna, una de las plataformas más sublimes en la obra de Mies, proyectada y construida durante los últimos años al frente del Departamento de Arquitectura del IIT, la encontramos en el edificio de oficinas Seagram, en Nueva York. El croquis frontal del edificio con el espacio de aproximación desde Park Avenue, desentendiéndose de la parte superior de fachada, representa magistralmente el protagonismo de la plataforma en una obra que, por sus intereses y por su ejecución, adquiere la condición de manifiesto. (FIG. 2) A los intereses del presente artículo, el Seagram anticipó unas propuestas urbanas sistemáticamente soportadas sobre plataformas que dotarían al proyecto de acceso y consistencia interna. [...]

A partir del Chicago Federal Center fue común que las plataformas contuvieran debajo de ellas cada vez más programa, pero no serían ya sino variaciones que harían del podio urbano un recurso tan eficaz como previsible. El último movimiento de la plataforma se produciría en Berlín, donde Mies construyera la Neue National Galerie. Siguiendo la taxonomía estructural que numerosos autores utilizan para clasificar sus obras, el proyecto de la Galería Nacional, comparte con Bacardi, y Schäfer, planta libre y tipología estructural: una cubierta cuadrada compuesta por una retícula ortogonal de jácenas sobre pilares perimetrales, retranqueados de los extremos.

En paralelo a esta idéntica solución del plano de la cubierta, Mies desarrolló tres propuestas totalmente distintas respecto a sus planos del suelo, reafirmando la tesis sobre el papel autónomo y prevalente de la plataforma, como elemento de mediación entre el edificio y el lugar, soporte físico y conceptual del sistema de acceso elevado de sus proyectos.

Si el relato de la Galería Nacional se explicara desde el plano del suelo, la secuencia debería empezar por la selección previa que Mies hace del emplazamiento, en detrimento de otras dos opciones propuestas por las autoridades berlinesas. Mies escogió el situado en el barrio de Tiergarten, ante la iglesia de San Mateo, de las pocas edificaciones que azarosamente se mantenía en pie tras la desolación de la guerra, en la esquina con la Potsdamerstrasse. [...]
Como operación urbana, Mies haría coincidir solar y plataforma, convertida así en el verdadero lugar del proyecto. Esto se haría especialmente patente en su planta definitiva, donde la aparentemente sencilla dislocación de su superficie cuadrada resolvía tangencialmente los accesos, al tiempo que regularizaba el contorno trapezoidal. A la recuperación de las correspondientes secuencias de aproximación oblicuas se superponían los accesos frontales característicos de la etapa americana.

El plano de la cubierta introducía otra excepción: retrasar el plano de fachada, construyendo un generoso porche hasta entonces monopolio de los edificios miesianos en altura, transposición literal de los cerramientos verticales del proyecto de Bacardí, desplazados por la climatología caribeña. La Galería Nacional rompía así una regla de oro mantenida desde el Campus del IIT según la cual los edificios de baja altura se entregarían uniformemente hasta el plano del suelo. Aquí, este desplazamiento del cerramiento reforzaba en alzado, la percepción del plano horizontal de la cubierta.

Más allá de las partes de programa que cada uno de los dos planos horizontales del proyecto cobijaría, la notable diferencia de longitud de trazo entre uno y otro desdice, una vez más, las recurrentes interpretaciones que vinculan las plataformas miesianas con el crepidoma de la arquitectura clásica, que se ajusta sistemáticamente a la proyección de la cubierta del templo que soportaba. Si acaso, el basamento miesiano establecería una más rigurosa vinculación con el Témenos de los santuarios. [...]

Arquitectónicamente Mies fija el proyecto a otro elemento urbano anclado en la memoria del lugar: la iglesia de San Mateo. Sobre el podio, en su parte posterior, la versión definitiva del proyecto ofrece un magnífico trompe l'oeil a través del cual la iglesia y, perpendicularmente a ella, el pabellón, descansan sobre el suelo de la plataforma, el témenos del lugar. (FIG. 5)

Una excepción más que Mies introdujo en el proyecto es la del recinto, que correspondia claramente a la etapa europea de Mies. Por el contrario, el equipaje arquitectónico que Mies se llevó hacia América no incluía el recinto murario que, como si hubiera sido un mero encofrado geométrico, dejó paso a la plataforma. La totalidad de la obra miesiana de aquella etapa americana estaría soportada por el podio. El recinto no reaparecerá hasta el proyecto para las oficinas Bacardí, en Cuba y, posteriormente, en la planta inferior de la Galería Nacional donde, como había sucedido en el Pabellón alemán de Barcelona, recinto y plataforma, patio y pabellón se reencontrarían.

Parecería que invocando de nuevo su presencia el maestro alemán hubiera querido cerrar un largo recorrido de cuarenta años, los que separaron la primera y la última gran plataforma de su obra. Pero si en Barcelona, plataforma y recinto coexistieron en planta, en Berlín los dos elementos sustantivos de su obra reclamaron de la sección. Abajo el recinto, arriba la plataforma. Al fondo, la iglesia de San Mateo.

El rellano/foyer del Crown Hall, correlato urbano del de la Farnsworth, fue no solo un episodio relevante por sí mismo, sino que afectó radicalmente a la ordenación definitiva del Campus. La limitación del escrito y la excepcionalidad tectónica del recurso, acotado a la década de los cincuenta, ha aconsejado mitirlo. En cualquier caso, en la tesis del autor La plataforma como umbral, se dedica a este episodio un extenso capítulo.

ABSTRACT A lo largo de su trayectoria profesional Mies mantuvo una
estricta observancia en relación a los dos planos horizontales que acotaron sistemáticamente su arquitectura, el del suelo y el de la cubierta, al punto de que sus atributos formales así como la interrelación que establecen definen en gran medida su arquitectura. El plano de la cubierta ha sido el que ha suscitado un mayor análisis y reconocimiento mientras que el otro plano horizontal, el del suelo, ha sido significativamente menos explorado a pesar de su indiscutible relevancia. En la arquitectura de Mies, la plataforma es sistemáticamente utilizada como un espacio de mediación con el lugar y a su vez ullizada como un espacio de mediación con el lugar y, a su vez, aproximación a su arquitectura. Plaxiorma y acceso constura.

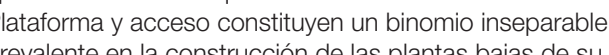
las plantas bajas de su arquitectura. La plataforma se convierte, por tanto, en el umbral de su arquitectura.

PALABRAS CLAVE: acceso elevado, límite, recinto, geometría.

JORDI ROS es arquitecto (ETSAB, 1983) y profesor titular del Departamento de Proyectos Arquitectónicos de la UPC. Actualmente es Director de la ETSAB. El artículo aborda parte de la investigación desarrollada en su tesis doctoral La plataforma como umbral. (ETSAB, 2013). 